Copyright 2013 IEEE. Published in the Annual International Conference of the IEEE Engineering in Medicine and Biology Society (EMBC - 2013), scheduled for July 03-07, 2013 in Osaka, Japan.

Personal use of this material is permitted.

However, permission to reprint/republish this material for advertising or promotional purposes or for creating new collective works for resale or redistribution to servers or lists, or to reuse any copyrighted component of this work in other works, must be obtained from the IEEE. Contact: Manager, Copyrights and Permissions / IEEE Service Center / 445 Hoes Lane / P.O. Box 1331 / Piscataway, NJ 08855-1331, USA. Telephone: + Intl. 908-562-3966

Note that this is a preprint, the final version of this paper can be found at IEEE Xplore 


\title{
A novel tracing method for the segmentation of cell wall networks
}

\author{
Jonas De Vylder ${ }^{1}$ and Filip Rooms ${ }^{1}$ and Stijn Dhondt ${ }^{2,3}$ and Dirk Inze ${ }^{2,3}$ and Wilfried Philips ${ }^{1}$
}

\begin{abstract}
Cell wall networks are a common subject of research in biology, which are important for plant growth analysis, organ studies, etc. In order to automate the detection of individual cells in such cell wall networks, we propose a new segmentation algorithm. The proposed method is a network tracing algorithm, exploiting the prior knowledge of the network structure. The method is applicable on multiple microscopy modalities such as fluorescence, but also for images captured using non invasive microscopes such as differential interference contrast (DIC) microscopes.
\end{abstract}

\section{INTRODUCTION}

Cells are the main building blocks of all organisms. Thus in order to understand the general working, aging and/or growing of organisms, it is of interest to study the cells and cell organization of these organisms. This is generally done using optical microscopy, since this allows visualising the cells in vivo. Visual inspection and manual processing of these microscopic images is still common in biological research. The process of annotating data is a tedious, time consuming and error prone task. To overcome this bottleneck in the microscopy pipeline, several automated methods based on computer vision have been investigated. An overview of different cell segmentation and tracking approaches can be found in [1], [2]. The majority of these methods start from generic segmentation strategies such as watershed, SVM classification or thresholding [3], [4], [5], [6].

The generic methods generally produce poor results, i.e. with under- or over-segmentation. This is due to clutter, bleed through, blur etc. To overcome these problems, an extra post-processing step is used to merge and/or split the segments in order to get more accurate segmentation results. This post-processing step is based on the intrinsic shape and appearance of the cells. Most of these methods perform well for images containing a sparse group of cells, but are hampered by dense clusters of cells such as is the case for organs where cells (not necessarily of the same type) are organized in a specific structure. More accurate and robust segmentation results are achieved using markers [7] or motion [8], however these approaches are less generic, limiting the applicability for specific applications.

*This research was carried out as part of the iMinds MMIQQA project http://www.iminds.be/en/research/overview-projects/p/detail/mmiqqa-2

${ }^{1}$ De Vylder J., Rooms F. . and Philips W. are with the department of Telecommunications and Information Processing, iMinds Image Processing and Interpretation Group, Ghent University jonas. devylderatelin.ugent. be

${ }^{2}$ Dhondt, S, and Inze, D. are with the department of Plant Systems Biology, VIB, 9052 Gent, Belgium

${ }^{3}$ Dhondt, S. and Inze, D. are with the department of Plant Biotechnology and Bioinformatics, Ghent University, 9052 Gent, Belgium
While many approaches in literature attempt to detect the cells directly, this work focuses on detecting the cells indirectly, by detecting the cell walls. This duality can help cell segmentation in situations where the cell walls form a network structure: all cells (and cell walls) touch each other and a cell cannot be enclosed by another cell. An example of such a network structure is shown in Fig. 2(a) where the cell walls of an Arabidopsis thaliana root are marked using a fluorescent marker.

In order to detect the cell wall network we propose the use of a tracing algorithm [9]. Several tracing algorithms have been proposed in the past. Two big groups of tracing techniques can be distinguished: global and local methods. Global methods first apply a segmentation step, e.g. based on thresholding, and then apply an analysis step where the segmentation result is processed, e.g. using morphological skeletonization, to a predefined structure such as a line or a network. The proposed method fits within the group of local methods, where a local neighbourhood is investigated in order to find parts of the segment; these new detected parts are then used to define new neighbourhoods to investigate. These methods iterate until no new parts of the segment are found. Local methods have been proposed in many forms: semi-automatic methods [10], [11], methods where the direction of the tracing line is predefined [12], [13], and random orientation tracing [14].

Especially the last method will be of interest for this work, since no prior knowledge on the direction of the cell walls is available. While the work in [14] focuses on detecting a single line, corresponding to the tail of a sperm cell, cell wall segmentation requires the detection of a network. In this paper we propose an extension of a random orientation tracing method which can cope with splitting and merging of lines, thus being well suited for network tracing.

This paper is arranged as follows. The next section provides a detailed description on tracing methods. First, a line tracing algorithm for random orientation is discussed. This is than extended to network tracing. Section III shows the results of our technique. Section IV recapitulates and concludes.

\section{SEGMENTATION USING TRACING}

\section{A. Line tracing}

In this subsection we will explain the tracing method described in [14], which can be seen as an intelligent subsampled version of [10]. This tracing method was developed to trace the tail of a single sperm cell, thus to trace a single line. In the next subsection we will extend this approach to network tracing. In order to trace a single line, we start 
from a seed point, $\mathbf{s}=(x, y)$. We look for a new point of the segment, i.e. the line, in a circular neighbourhood:

$$
N_{r}=\left\{\mathbf{n} \mid \mathbf{n} \in \Omega \wedge \operatorname{round}\left(\|\mathbf{n}-\mathbf{s}\|_{2}\right)=r\right\}
$$

with $\Omega=[0, K] \times[0, L]$ and $K \times L$ the dimension of the image. The pixel in this neighbourhood that has the strongest intensity, and that does not correspond to a pixel which was a seed in the past, nor lies in the vicinity of previous seeds, will be chosen as the next seed, i.e. the next point in the traced line. This process is iterated until all points in the circular neighbourhood belong to one of the following groups:

1) the point has been a seed point

2) the point lies in the vicinity of a seed point

3) the point has an intensity less than a predefined threshold

We illustrate this tracing step using Fig. 1.B-C. In Fig. 1.B an image is shown, where the seed point is marked using a green square, and where all the pixels belonging to the circular neighbourhood are shown in red. This circular neighbourhood is also shown in 1.C, where all pixels not belonging to the neighbourhood are set to black. For this specific neighbourhood, 4 regions correspond to values above the threshold (marked as a-d). If this is the first iteration, i.e. if no pixel has been a seed in the past, then the white pixel in region $\mathrm{c}$ will be chosen as next seed point. However if this is not the first iteration and if that pixel was the previous seed point, then we have to remove the complete region as candidate seed point. Then the white pixel in region $b$ will have the highest intensity, thus becoming the next seed point.

\section{B. Network tracing}

The line tracing algorithm is useful for applications where a small number of lines have to be traced, e.g. sperm tail detection in microscopic images, but is unsuitable for applications with a random number of lines with bifurcations, such as is the case in cell wall networks (Fig. 1.A). In order to extend the tracing algorithm to networks, we also start from a seed, $\mathbf{s}$, and a circular neighbourhood $N_{r}$, but the future seed selection procedure will be handled in an iterative way. First, the values of all pixels in the neighbourhood which already belong to the segment, or are in the vicinity of a segment pixel, are set to zero. Then the pixel with the highest value is chosen as a potential seed point, $\mathbf{s}_{p}$. If the value of $\mathbf{s}_{p}$ is above a threshold, the point is added to the set of future seed points, $F$. All points on the line between $\mathbf{s}$ and $\mathbf{s}_{p}$ are added to the segment pixels. In real applications, a line typically is more than one pixel width, thus to avoid backtracking in the tracing algorithm, we also add all the pixels within a small distance, $t_{d}$, to the line segment. So the new set of segment pixels is defined as:

$$
\begin{aligned}
D_{i+1}= & D_{i} \cup\left\{\mathbf{x}_{\mathbf{2}} \mid \exists \alpha \in[0,1]:\right. \\
& \left.\left(\left(\mathbf{x}_{\mathbf{1}} \triangleq \alpha \mathbf{s}+(1-\alpha) \mathbf{s}_{p}\right) \wedge\left(\left\|\mathbf{x}_{\mathbf{1}}-\mathbf{x}_{\mathbf{2}}\right\|_{2}<t_{d}\right)\right)\right\}
\end{aligned}
$$

with $D_{i}$ the set of segment pixels before adding the new seed.
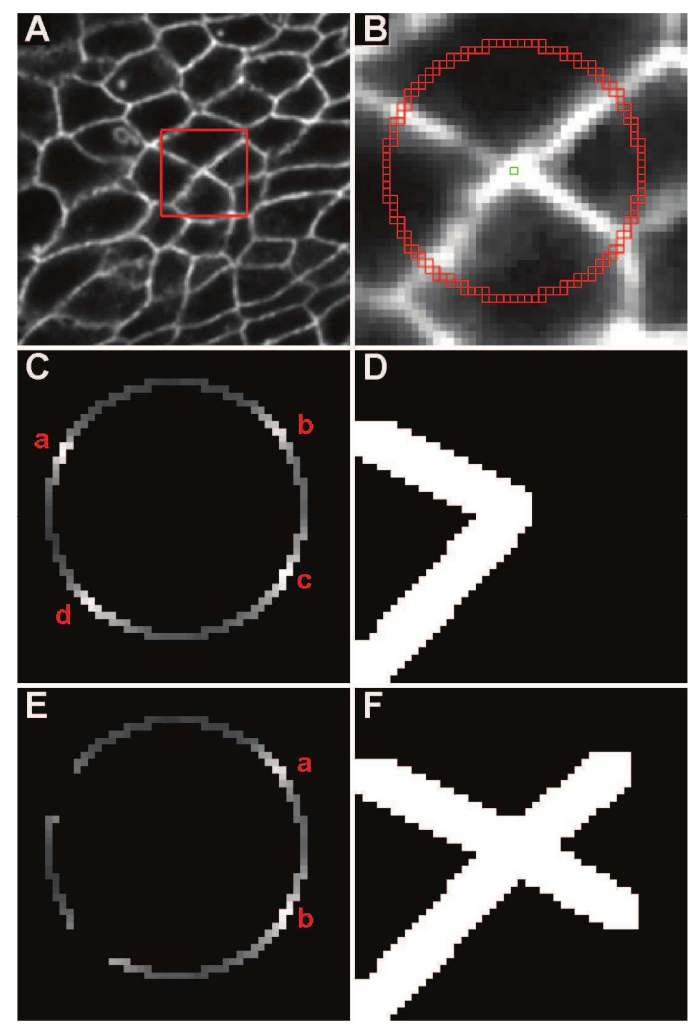

Fig. 1. An overview of the workflow of the network tracing algorithm. (A) a microscopic image where cell walls are tagged using fluorescent markers. (B) A close up of the red square in (A). The seed is shown in green and the circular neighbourhood is delineated in red. (C) The intensities of the circular neighbourhood. (D) the detected pixels at the start of processing the seed. (E) The circular neighbourhood with the detected pixels removed. (F) the detected pixels at the end of processing the seed.

This process is now iterated, i.e. a new potential seed is detected (note that the value corresponding to the seed that has just been added, is set to zero since it now belongs to the segment), which will be added if it has a higher value than a predefined threshold. This iterative process stops if no points in the neighbourhood have a value above the threshold, this seed point will be added to the end point set, $E$. After finding an end point, a new seed from the future seed set, $F$, is chosen as seed, thus removing it from $F$. For this new seed a new circular neighbourhood will be calculated, and new potential seed points will be searched. This iterative seed point selection ends if there are no points remaining in the future seed set $F$. Note that since points cannot be connected to an already detected pixel, that the end points (the points in $E$ ) are only connected to the network as a line. In order to connect the end of the line with the rest of the network, the line tracing algorithm described in subsection II-A can be used.

An example of the work flow is shown in Fig. 1. A cell wall network, visualized using fluorescent markers is shown in Fig. 1.A. A close up of the red square is shown in Fig. 1.B, where a seed point is delineated in green and the circular neighbourhood in red. For clarity the circular neighbourhood is also shown in Fig. 1.C, where all other pixel values are 
set to zero. Note that in this neighbourhood, there are 4 regions which correspond to a value higher than the set threshold (denoted with a-d). If Fig. 1.D is a visualization of the already detected pixels, than the values corresponding to segment in the neighbourhood are set to zero, which results in Fig. 1.E. After two iterations all possible seed points, a and $b$, are found, resulting in the segment shown in Fig. 1.F. The found seeds, $a$ and $b$ will be processed in future steps.

\section{Border tracing}

The proposed tracing method is able to trace networks of bright lines on a dark background. This assumption is suitable for a fluorescent micrograph where everything lies in the same focal plane, but clearly restricts the applicability of the algorithm. For example if the intensity changes over the image ( e.g. Fig. 2), it becomes difficult to set a good threshold for the seed points. Therefore we propose to first pre-process the images to enhance the network structure. The filters used for these can change on the application. For fluorescent microscopy images a ridge filter is recommendable [15], for wide-field microscopy standard edge filters [16] will show good results and for differential interference contrast (DIC) microscopy specially designed DIC edge filters will greatly improve the results [17], [18].

\section{RESULTS}

In a first example we test the proposed method on the segmentation of plant cells in A. thaliana roots. The cell walls are marked using a fluorescent dye. The cells are organized in a structure intrinsic to the root, resulting in a cell wall network, as can be seen in Fig. 2.(a). To partially remove the non-uniform intensity of the dye, we pre-process the image using a Frangi-ridge filter [15]. The result using different methods is shown in Fig. 2.(b-d). Figure 2.(b) is the result of a watershed based segmentation [2]. This results in many small false segments located at the cell borders. Fig. 2.(c) corresponds to the result of a global tracing method [9], which also results in over segmentation. The proposed method generally detects the cell walls correctly (Fig. 2.(d)). Note that the Frangi-ridge pre-processing step is insufficient to remove extreme situations of variable intensities. Therefore the cell walls with very low intensity (the cells at the right top of the image) are not detected with any of the methods. This might be overcome by local intensity correction [19], at the risk of blowing up the noise at certain locations.

We also test the proposed method using a second use case: the analysis of DIC microscopic images. These images are of interest for biologists, since this imaging modality is noninvasive, in contrast to fluorescence microscopy. However, this image modality raises many challenges for generic state of the art segmentation techniques [18]. An example of such an image is shown in Fig. 3(a). To overcome the lack of contrast in specific directions, multiple images (each with contrast in a different direction) are combined in the Danoso edge filter [18]. The result of the proposed network segmentation technique is shown in Fig 3.(b). It is clear

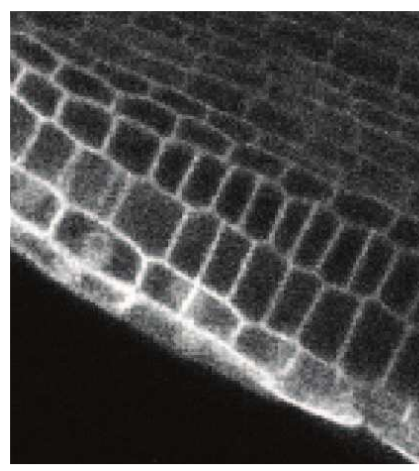

(a) RAW image

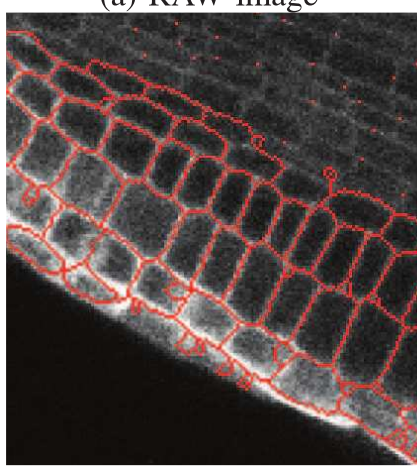

(c) global tracing

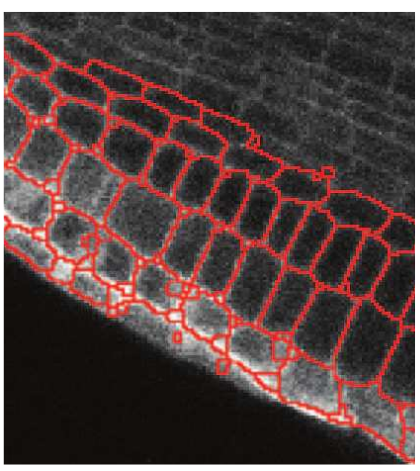

(b) watershed

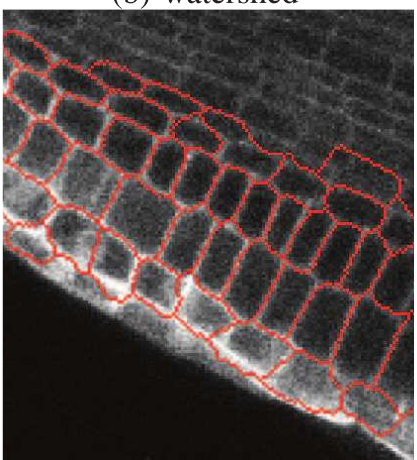

(d) proposed processing
Fig. 2. An example of cell detection in the root tip of A. thaliana. At the left the original microscopic image, where cell walls are tagged using fluorescent markers. At the right the result of the proposed method is shown.

that the method works well for the detection of pavement cells, i.e. the big puzzle shaped cells (delineated in red). The detection of the stomata (delineated in green) is a bit less accurate. This is because the stomata actual consists of two cells: guard cells. Sometimes both guard cells are detected, while for one stomata only one segment was found. However this is less of an issue, since the stomata have a specific size and shape, allowing to merge the guard cells in to single stomata in a post-processing step.

This segmentation result is quantitatively compared with the results using the work from[18], which is as far as the authors are aware the only other method developed for the segmentation of A. thaliana cells in DIC images. The validation is done in comparison with manual annotated ground truth. The segmentation quality is expressed in terms of correct segmentation (C), over segmentation (O) and under segmentation $(\mathrm{U})$ : i.e. respectively the amount of multiple segments that where found corresponding to a single cell and the amount of multiple cells corresponding to a single segment. The results are shown in Table I. Note that pavement cells are generally better detected than stomata. The over segmentation in the proposed method is only due to the detection of individual guard cells, which is normal since they have the same edge properties as pavement cells. The correct detection of guard cells will make it easier to reconstruct the stomata, since there organization is well known. The method discussed in [18] does not have this 


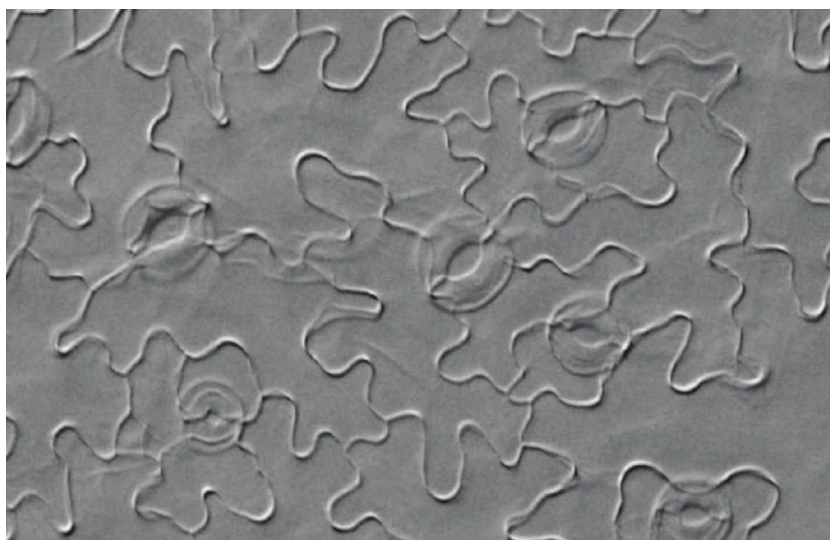

(a) RAW image

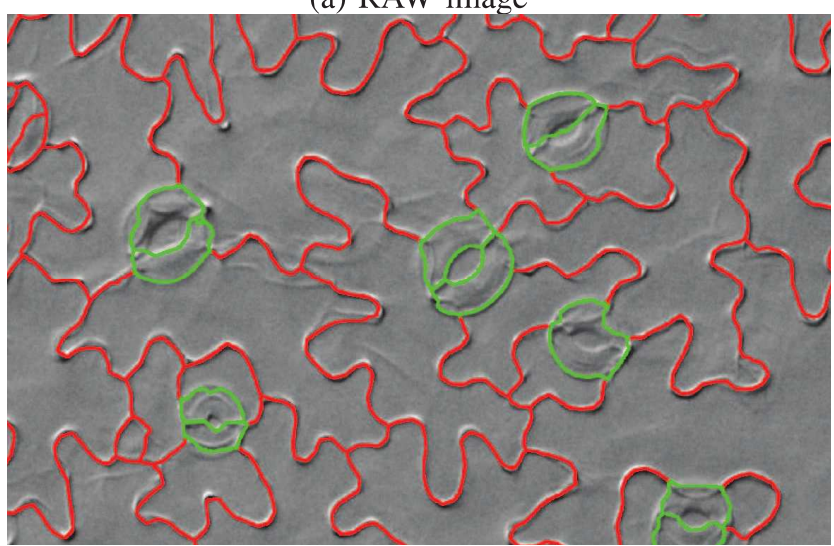

(b) proposed processing

Fig. 3. An example of cell detection in Arabidopsis leaves imaged using DIC microscopy. At the top the original DIC microscopic image. At the bottom the result of the proposed method is shown. Pavement cells are delineated in red, stomata in green.

TABLE I

QUANTITATIVE RESULTS FOR THE SEGMENTATION OF DIC IMAGES

\begin{tabular}{|ll|ccc|}
\hline method & cell type & C & O & U \\
\hline$[18]$ & pavement cells & 19 & 3 & 1 \\
proposed & pavement cells & 21 & 0 & 0 \\
{$[18]$} & stomata & 0 & 5 & 3 \\
proposed & stomata & 3 & 0 & 4 \\
\hline
\end{tabular}

advantage, the over segmentation there is completely random and is not due to the presence of guard cells.

\section{CONCLUSIONS}

This paper proposes a novel tracing algorithm for network structures. The proposed method can be used for the segmentation of dense cell clusters, where the cell walls are seen as a network. The method is generic in the sense that it can be used for different microscopy modalities. The method was tested for the segmentation of cells in fluorescent microscopy images and differential interference contrast. The method was validated for the detection of pavement cells and stomata. The proposed method showed more accurate results for both type of objects than state of the art segmentation methods.

\section{REFERENCES}

[1] L. Shamir, J. D. Delaney, N. Orlov, D. M. Eckley, and I. G. Goldberg, "Pattern recognition software and techniques for biological image analysis," PLOS Computational Biology, vol. 6, no. 11, 2010.

[2] E. Meijering, "Cell segmentation: 50 years down the road," IEEE Signal Processing Magazine, vol. 29, no. 5, pp. 140-145, 2012.

[3] C. Ruberto, A. Dempster, S. Khan, and B. Jarra, "Analysis of infected blood cell images using morphological operators," Image and Vision Computing, vol. 20, pp. 133-146, 2002.

[4] M. Marcuzzo, P. Quelhas, A. Campilho, A. M. Mendonca, and A. Campilho, "Automated arabidopsis plant root cell segmentation based on svm classification and region merging," Computers in Biology and Medicine, vol. 39, no. 9, pp. 785-793, 2009.

[5] L. Kamentsky, T. R. Jones, A. Fraser, M. A. Bray, D. J. Logan, K. L. Madden, V. Ljosa, C. Rueden, K. W. Eliceiri, and A. E. Carpenter, "Improved structure, function and compatibility for cellprofiler: modular high-throughput image analysis software," Bioinformatics, vol. 27, no. 8, pp. 1179-1180, 2011.

[6] M. P. Pound, A. P. French, D. M. Wells, M. J. Bennett, and T. P. Pridmore, "Cellset: Novel software to extract and analyze structured networks of plant cells from confocal images," Plant Cell, vol. 24, no. 4, pp. 1353-1361, 2012.

[7] P. Quelhas, M. Marcuzzo, A. M. Mendonca, and A. Campilho, "Cell nuclei and cytoplasm joint segmentation using the sliding band filter," IEEE Transactions on Medical Imaging, vol. 29, no. 8, pp. 1463-1473, 2010.

[8] T. Roberts, S. McKenna, N. Wuyts, T. Valentine, and A. Bengough, "Performance of low-level motion estimation methods for confocal microscopy of plant cells in vivo," in Motion and Video Computing, 2007. WMVC '07. IEEE Workshop on, 2007, pp. 13-13.

[9] E. Meijering, "Neuron tracing in perspective," Cytometry Part A, vol. 77A, no. 7, pp. 693-704, 2010.

[10] E. Meijering, M. Jacob, J. C. F. Sarria, P. Steiner, H. Hirling, and M. Unser, "Design and validation of a tool for neurite tracing and analysis in fluorescence microscopy images," Cytometry Part A, vol. 58A, no. 2, pp. 167-176, 2004

[11] A. Jørgensen, S. Schmidt, N.-H. Staalsen, and L. Østergaard, Semiautomatic vessel tracking and segmentation using epicardial ultrasound in bypass surgery, ser. IEEE Engineering in Medicine and Biology Society. Conference Proceedings. IEEE Press, 2012, pp. 2331-2334.

[12] C. M. Sun, P. Vallotton, D. D. Wang, J. Lopez, Y. Ng, and D. James, "Membrane boundary extraction using circular multiple paths," Pattern Recognition, vol. 42, no. 4, pp. 523-530, 2009.

[13] A. French, S. Ubeda-Tomas, T. J. Holman, M. J. Bennett, and T. Pridmore, "High-throughput quantification of root growth using a novel image-analysis tool," Plant Physiology, vol. 150, no. 4, pp. 1784-1795, 2009.

[14] A. Bijar and M. Mikaeili, "Sperm's tail identification and discrimination in microscopic images of stained human semen smear," in Image and Signal Processing and Analysis (ISPA), 2011 7th International Symposium on, 2011, pp. 709-714.

[15] A. F. Frangi, W. J. Niessen, R. M. Hoogeveen, T. van Walsum, and M. A. Viergever, "Model-based quantitation of 3-d magnetic resonance angiographic images," IEEE Transactions on Medical Imaging, vol. 18, no. 10, pp. 946-956, 1999.

[16] Gonzales and Woods, Digital image processing, 3rd ed. Upper Saddle River, N.J.: Prentice Hall, 2008, 2009289249Rafael C. Gonzalez, Richard E. Woods.ill. (some col.) ; $25 \mathrm{~cm}$.Includes bibliographical references (p. 915-942) and index.

[17] F. Kagalwala and T. Kanade, "Reconstructing specimens using dic microscope images," IEEE Transactions on Systems Man and Cybernetics Part B-Cybernetics, vol. 33, no. 5, pp. 728-737, 2003.

[18] D. Ochoa Donoso, "Biological image analysis of model organisms," Ph.D. dissertation, Ghent University. Faculty of Engineering and Architecture, 2011.

[19] J. Charles, B. Kuncheva, L.and Wells, and I. Lim, "Background segmentation in microscope images," in Proc 3rd International Conference on Computer Vision Theory and Applications VISAPP08, 2008. 